\title{
Numerical Modelling of Round Impinging Jet Heat Transfer Using Scale-Resolving-Simulation Methods: Influence of Local Grid Refinement
}

\author{
Tomáš Koř́nek ${ }^{1}$, and Tomáš Tisovský ${ }^{1}$ \\ ${ }^{1}$ Technical University of Liberec, Department of Power Engineering Equipment, Studentská 2, 46117 \\ Liberec, Czech Republic
}

\begin{abstract}
An accurate prediction of heat transfer is crucial in many engineering applications and a typical representative is heat transfer of impinging jets. The present study addresses numerical simulations using three Scale-Resolving-Simulation methods, namely the Detached Eddy Simulation, Large Eddy Simulation and the Partially Averaged NavierStokes. The study focus on the influence of a local grid refinement on predicted heat transfer. The closest match with experimental data was achieved with DES and PANS $(f k=0.5)$ models for all computational grids. The grid refinement led to an improvement of predicted results for LES and PANS with a high physical resolution $(f k=0.25)$.
\end{abstract}

\section{Introduction}

Complex geometries are common in many engineering applications and a generation of structured computational grids is cumbersome on such domains. The computational grids with very fine near wall resolution are typically required to correctly predict turbulent heat transfer from a wall. This increase of grid points results in a higher demand on computational resources.

Two ways are common to reduce amount of grid points, a use of wall functions or a local grid refinement. The use of the first named is questionable as wall functions are not applicable in all regions for example in case where a flow separation occurs [1]. The second method, the local grid refinement, is available in the most grid generators and is easy to use. The local grid refinement is then applied in areas where the higher spatial resolution is required [2,3]. However, a rapid change of a grid size could have a negative effect on predicted results for the grid dependent turbulent models such as Scale-Resolving-Simulation (SRS) models.

Heat transfer and fluid dynamics of the round impinging jet have been extensively studied for various Reynolds numbers and nozzle-plate distances [4-8]. Impinging jets involve several flow dynamics mechanisms: stagnation, separation, reattachment etc. Such complex flow dynamics along with a large amount of experimental and numerical simulations data of

\footnotetext{
${ }^{1}$ Corresponding author: tomas.korinek@tul.cz
} 
this canonical case make it the perfect test case to validate a new turbulent models, boundary conditions, numerical schemes and other techniques used in computational fluid dynamics.

The present study aims on a comparison of SRS methods and various strategies for a grid refinement on the heat transfer of the round impinging jet at Reynolds number $R e=23000$ (based on the nozzle diameter, the inlet velocity and kinematic viscosity) and the nozzle-plate distance $H / D=6$. Results from the conducted study have been compared against various experimental data [6-8].

\section{Mathematical model}

Filtered continuity and momentum equations based on the instantaneous velocity decomposition $V_{\mathrm{i}}=U_{\mathrm{i}}+u_{\mathrm{i}}$ are:

$$
\begin{gathered}
\frac{\partial \mathrm{U}_{i}}{\partial \mathrm{x}_{i}}=0 \\
\frac{\partial \mathrm{U}_{i}}{\partial \mathrm{t}}+\mathrm{U}_{j} \frac{\partial \mathrm{U}_{i}}{\partial \mathrm{x}_{j}}=-\frac{\partial \mathrm{p}}{\rho \partial \mathrm{x}_{i}}+v \frac{\partial^{2} U_{i}}{\partial \mathrm{x}_{j} \partial \mathrm{x}_{j}}-\frac{\partial \tau\left(V_{i}, \mathrm{~V}_{j}\right)}{\partial \mathrm{x}_{j}}
\end{gathered}
$$

where $V_{\mathrm{i}}$ is the instantaneous velocity, $U_{\mathrm{i}}$ is the filtered velocity, $u_{\mathrm{i}}$ is the unresolved/subfilter velocity, $p$ is the filtered pressure, $v$ is the kinematic viscosity and $\tau\left(V_{\mathrm{i}}, V_{\mathrm{j}}\right)$ is the generalized second moment which correspond to the effect of the unresolved motion to the resolved one and is calculated as

$$
\tau\left(V_{i}, V_{j}\right)=-2 v_{u} S_{\mathrm{ij}}+\frac{2}{3} k_{u} \delta_{\mathrm{ij}}, S_{\mathrm{ij}}=\frac{1}{2}\left(\frac{\partial \mathrm{U}_{i}}{\partial \mathrm{x}_{j}}+\frac{\partial \mathrm{U}_{j}}{\partial \mathrm{x}_{i}}\right),
$$

where subscript $u$ denotes an unresolved/sub-filter quantity. The simplified filtered energy equation is in form

$$
\frac{\partial \mathcal{T}}{\partial \mathrm{t}}+\mathrm{U}_{j} \frac{\partial \mathcal{T}}{\partial \mathrm{x}_{j}}=\frac{\partial}{\partial \mathrm{x}_{j}}\left(\frac{v}{\operatorname{Pr}} \frac{\partial \mathcal{T}}{\partial \mathrm{x}_{j}}\right)-\frac{\partial \tau\left(\mathrm{T}, \mathrm{V}_{j}\right)}{\partial \mathrm{x}_{j}}
$$

where $T$ is the filtered temperature, $\operatorname{Pr}$ is Prandtl number and $\tau\left(T, V_{\mathrm{j}}\right)$ is calculated from

$$
\tau\left(\mathrm{T}, \mathrm{V}_{j}\right)=-\frac{v_{u}}{\mathrm{Pr}_{t}} \frac{\partial \mathrm{T}}{\partial \mathrm{x}_{j}}
$$

where $P r_{t}$ is turbulent Prandtl number ( $P r_{t}=0.85$ in the present study).

\subsection{Large Eddy Simulation model}

The Large Eddy Simulation (LES) method with the sub-grid scale (SGS) model WALE [9] was one of the SRS methods used to close the system of filtered equations. The SGS model has advantages over the Smagorinsky model as naturally damp the eddy viscosity near walls and does not require additional damping [10]. 


\subsection{Detached Eddy Simulation model}

The second tested SRS method is the Detached Eddy Simulation (DES) method. It is a hybrid RANS/LES model [11] where the switch between LES and RANS is based on the length scale. If the turbulent length scale is larger than the local grid size, the model operates in the LES mode, otherwise it operates in the RANS mode. The DES model used in the present study is based on the k- $\omega$ SST RANS model [12].

\subsection{Partially-Averaged Navier-Stokes model}

The Partially-Averaged Navier-Stokes (PANS) model [13] is based on the partially-averaged turbulent kinetic energy $k_{\mathrm{u}}$ and its dissipation rate $\varepsilon_{\mathrm{u}}$ or unresolved specific eddy dissipation rate $\omega_{\mathrm{u}}$. Relations between total and partially averaged (unresolved) is controlled by two parameters, namely the ratio of unresolved to total turbulent kinetic energy $f_{k}$ and the ratio of unresolved to total dissipation rate.

$$
f_{k}=\frac{k_{u}}{k}, f_{\varepsilon}=\frac{\varepsilon_{u}}{\varepsilon}, f_{\omega}=\frac{\omega_{u}}{\omega}=\frac{f_{\varepsilon}}{f_{k}} .
$$

The present study uses the PANS k- $\omega$ TNT model [14], where the unresolved turbulent viscosity is calculated from

$$
v_{u}=\frac{k_{u}}{\omega_{u}} .
$$

The governing equations of the PANS k- $\omega$ TNT model are

$$
\begin{gathered}
\frac{\partial \mathrm{k}_{u}}{\partial \mathrm{t}}+\mathrm{U}_{j} \frac{\partial \mathrm{k}_{u}}{\partial \mathrm{x}_{j}}=\mathrm{P}_{k}-\beta \mathrm{k}_{u} \omega_{u}+\frac{\partial}{\partial \mathrm{x}_{j}}\left[\left(v+\frac{v_{u} f_{\omega}}{\sigma_{k} f_{k}}\right) \frac{\partial \mathrm{k}_{u}}{\partial \mathrm{x}_{j}}\right] \\
\frac{\partial \omega_{u}}{\partial \mathrm{t}}+\mathrm{U}_{j} \frac{\partial \omega_{u}}{\partial \mathrm{x}_{j}}=\frac{\alpha}{v_{u}} P_{k}-\left(P^{\prime}-\frac{P^{\prime}}{f_{\omega}}+\frac{\beta \omega_{u}}{f_{\omega}}\right) \omega_{u}+\frac{\partial}{\partial \mathrm{x}_{j}}\left[\left(v+\frac{v_{u} f_{\omega}}{\sigma_{\omega} f_{k}}\right) \frac{\partial \omega_{u}}{\partial \mathrm{x}_{j}}\right] \\
+\sigma_{D} \frac{1}{\omega} \frac{f_{\omega}}{f_{k}} \frac{\partial \mathrm{k}_{u}}{\partial \mathrm{x}_{j}} \frac{\partial \omega_{u}}{\partial \mathrm{x}_{j}}, \\
\mathrm{P}^{\prime}=\frac{\alpha \beta \mathrm{k}}{v_{u}}, P_{k}=v_{u}\left(\frac{\partial \mathrm{U}_{i}}{\partial \mathrm{x}_{j}}+\frac{\partial \mathrm{U}_{j}}{\partial \mathrm{x}_{i}}\right) \frac{\partial \mathrm{U}_{i}}{\partial \mathrm{x}_{j}}
\end{gathered}
$$

where $\alpha, \beta, \beta^{*}, \sigma_{\mathrm{D}}, \sigma_{\mathrm{k}}, \sigma_{\omega}$ are model constant same as in the original k- $\omega$ TNT RANS model by Kok [15].

\section{Computational setup}

The computational domain was parametrized using the nozzle diameter D. A schematic view on the computational domain is shown on Figure 1. The inlet was located 6 diameters above the heated wall. Three different conditions were used for the upper part of the domain, namely a slip wall, no slip wall and outflow. 


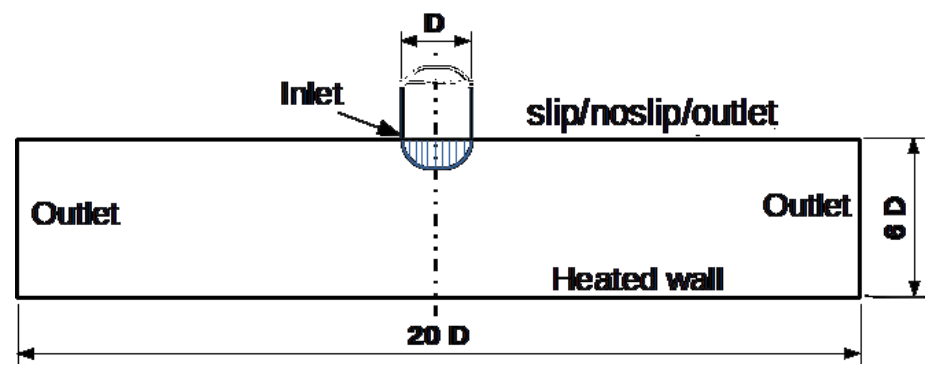

Fig. 1. A schematic view on the computational domain.

Inlet velocity, turbulent kinetic energy and specific dissipation rate profiles were obtained from a pipe flow simulation at $\mathrm{Re}=23000$ based on the pipe diameter, bulk velocity and kinematic viscosity. Additionally, the inlet velocity was perturbed with random fluctuations with the turbulence intensity IT $=5 \%$.

A hexahedral block structured computational grid was generated in ANSYS ICEMCFD software and contained 0.5 million of computational cells. Two additional computational grids were created by a refinement of the original grid. The first refinement was located only near the heated wall to the height 1 diameter above the wall and 2 diameters from the axis of the nozzle. For the second refined grid, the refinement was done on the whole height of the domain (from the nozzle to the heated wall). Parameters of the generated computational grids are in Table 1 and a detail of the grids is on Figure 2.

Table 1. Computational grids.

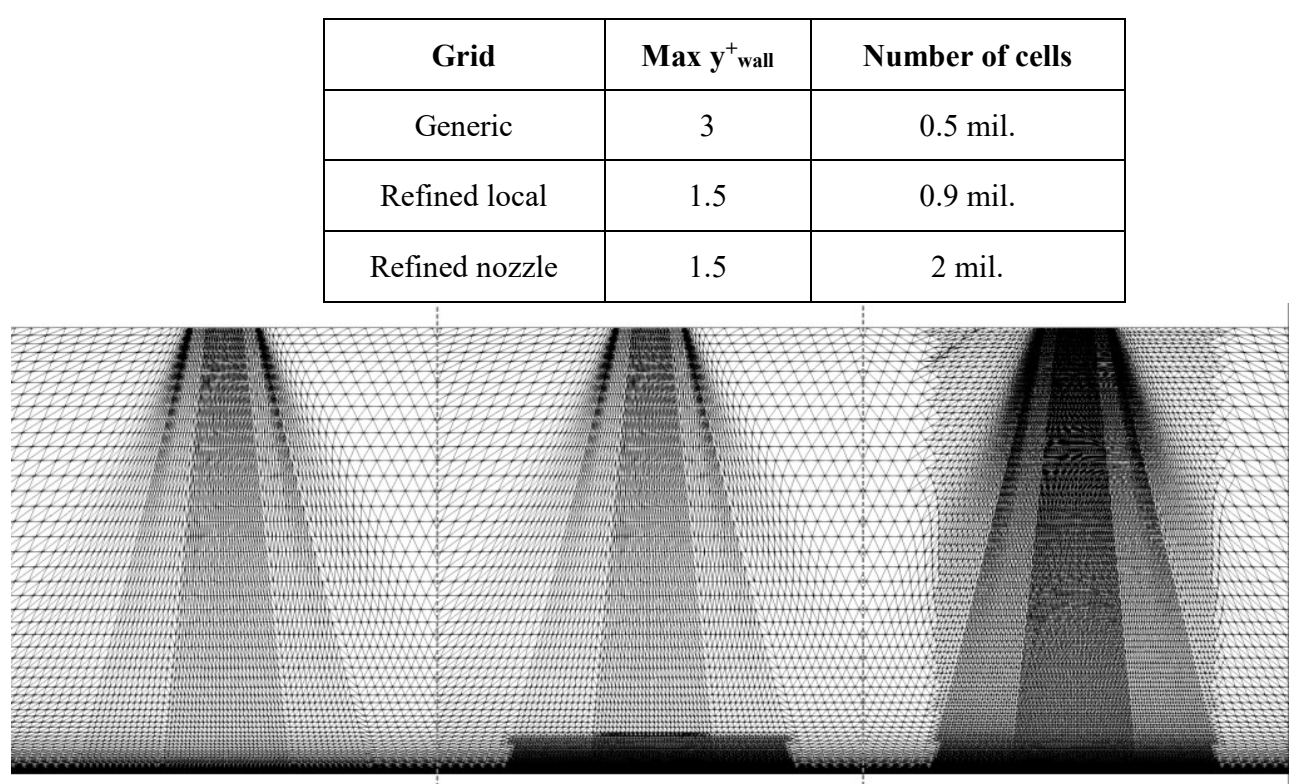

Fig. 2. A detail on computational grids near the nozzle and heated wall.

A choice of an optimal physical resolution is crucial for the PANS model. At first, the physical resolution has to be supported by the computational grid for the correct behavior of the model. In contrary, a too high physical resolution results in a high eddy viscosity which leads to a damping of introduced perturbation. The probability density function (PDF) of the $\mathrm{f}_{\mathrm{k}}$ parameter in the domain obtained from the RANS precursor simulation is shown 
in Figure 3. The $f_{k}$ parameter is computated using the expression introduced by Foroutan and Yavuzkurt [16]. Obviously, a grid refinement led to a decrease of the optimal $f_{k}$ parameter as a result of the decreased local grid size.

Based on the precursor simulation, two $f_{k}$ parameters (physical resolutions) for PANS simulation were tested, namely a high physical resolution $f_{k}=0.25$ and a moderate physical resolution $f_{k}=0.5$. The second parameter $f_{\varepsilon}$ is set to unity as the use of a lower value led to a worse predcitions [17].

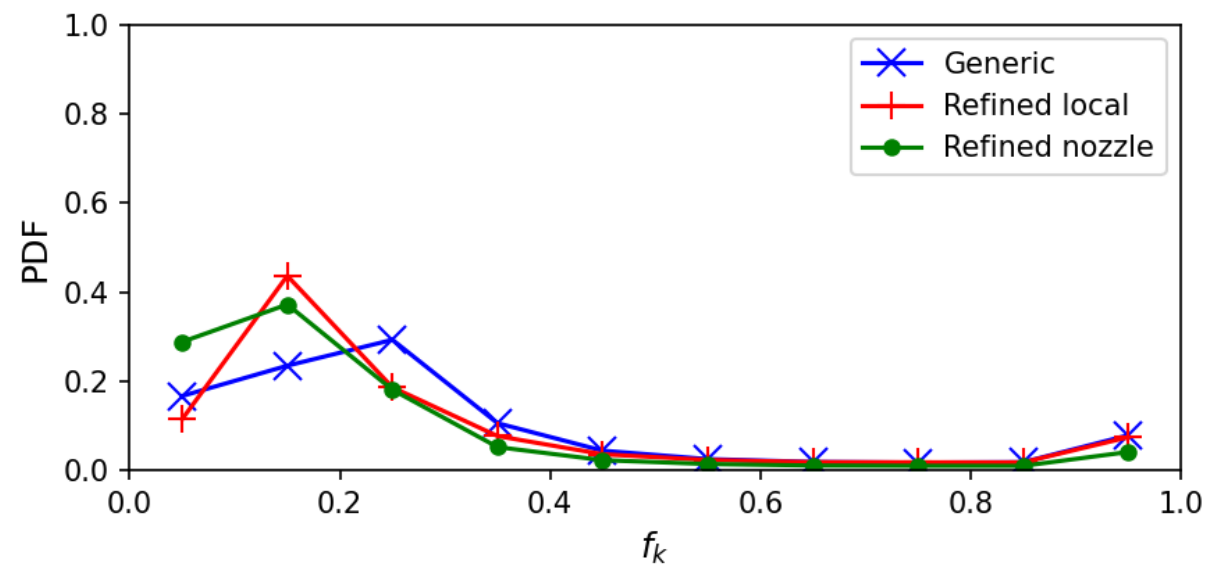

Fig. 3. Probability Density Function of the physical resolution represented by the $f_{k}$ parameter.

\section{Results}

Time averaged Nusselt number $(\mathrm{Nu})$ distributions obtained from numerical simulations are shown on Figure 4. The closest match with the experimental data was for DES model and PANS with $f_{k}=0.5$, especially near the stagnation point. In contrary, RANS over-predicted heat transfer near the stagnation point. The over-prediction is due a slow decay of the mean velocity profile along the nozzle axis. Other SRS models, LES and PANS $f_{k}=0.25$, were in a good agreement with experimental data up to 1 diameter from the nozzle axis. However, further downstream an under-prediction appeared in this region. It seems, that the coarse spatial resolution far from the nozzle axis resulted in a poor prediction of near wall turbulent structures whose have a great effect on the heat transfer from the wall. Similar was observed in impinging jets by Hadziabdic and Hanjalic [18] for LES and more recently by Kubacki and Dick [19] for hybrid RANS/LES simulations. Additionally, simulations on a coarse grid with a high physical resolution can lead to a worse prediction of heat transfer. This behavior was observed for PANS at the backward facing step flow [20]. 


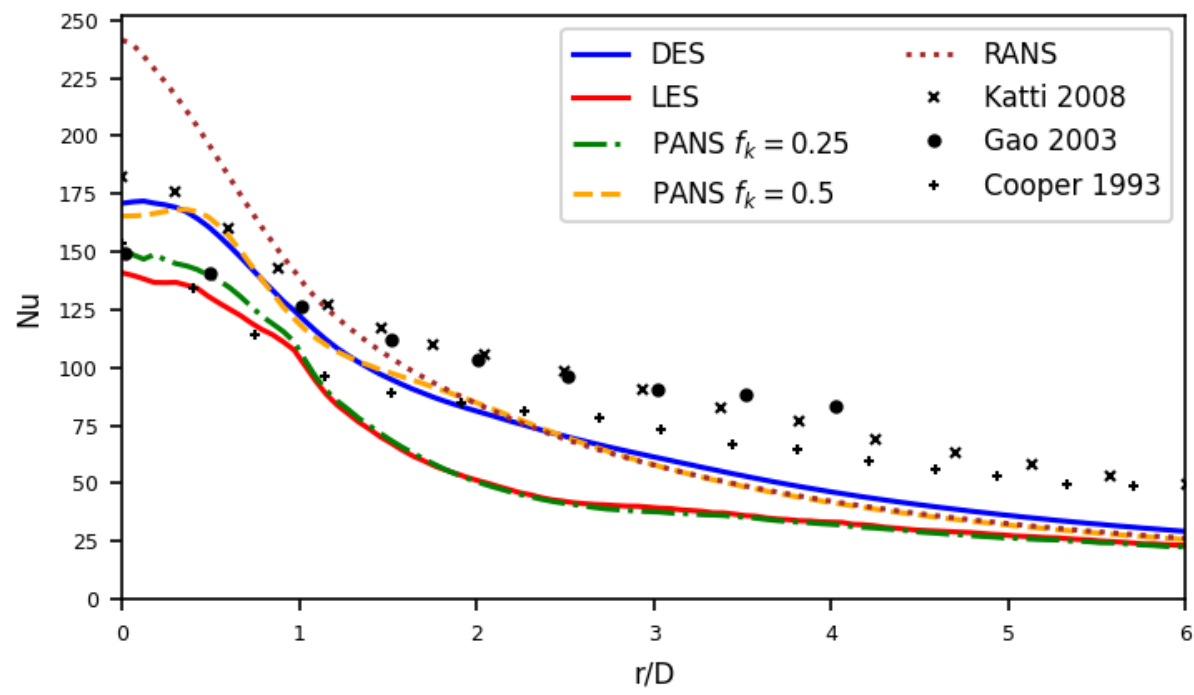

Fig. 4. Nusselt number distribution on the heated wall for various turbulent models.

The second part of the present study focused on an influence of a grid refinement on the predicted heat transfer. The distribution of the time-averaged Nusselt number on the heated wall is shown on Figure 5 for DES and LES and on Figure 6 for PANS. Only a slight change in Nusselt number distributions among grids can be observed for DES. In contrary, the grid refinement led to an increase of the Nusselt number near the stagnation point for LES and same was for PANS $f_{k}=0.25$.
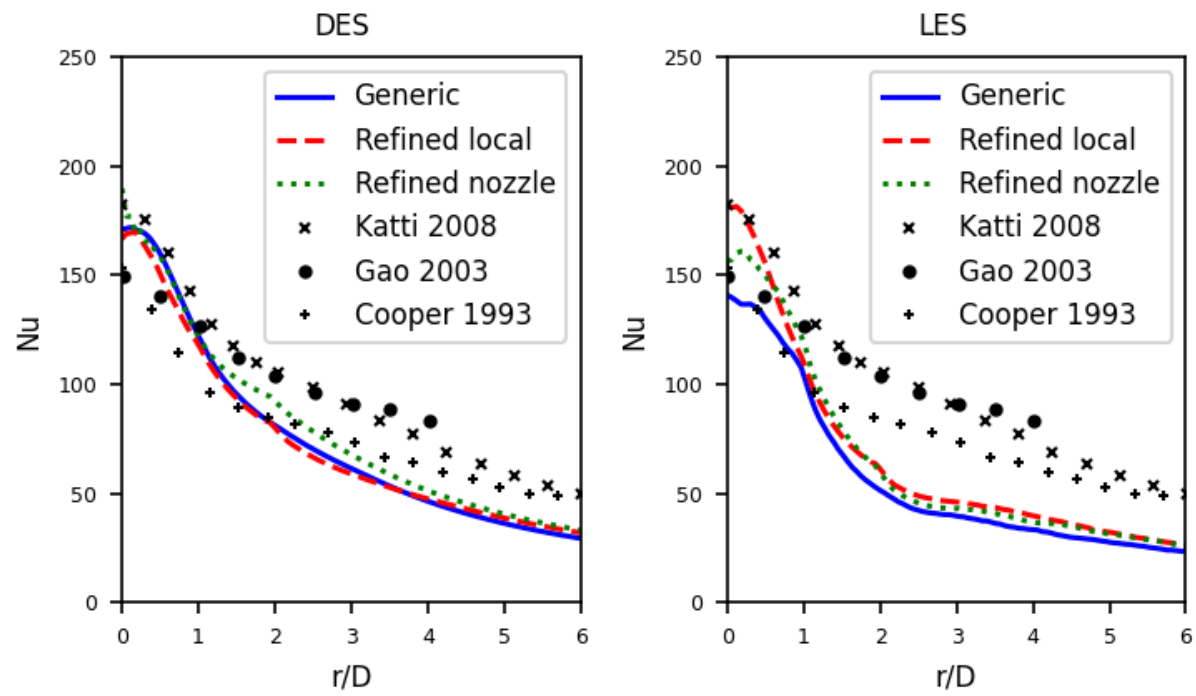

Fig. 5. An influence of the grid refinement on the Nusselt number distribution on the heated wall for DES and LES models. 

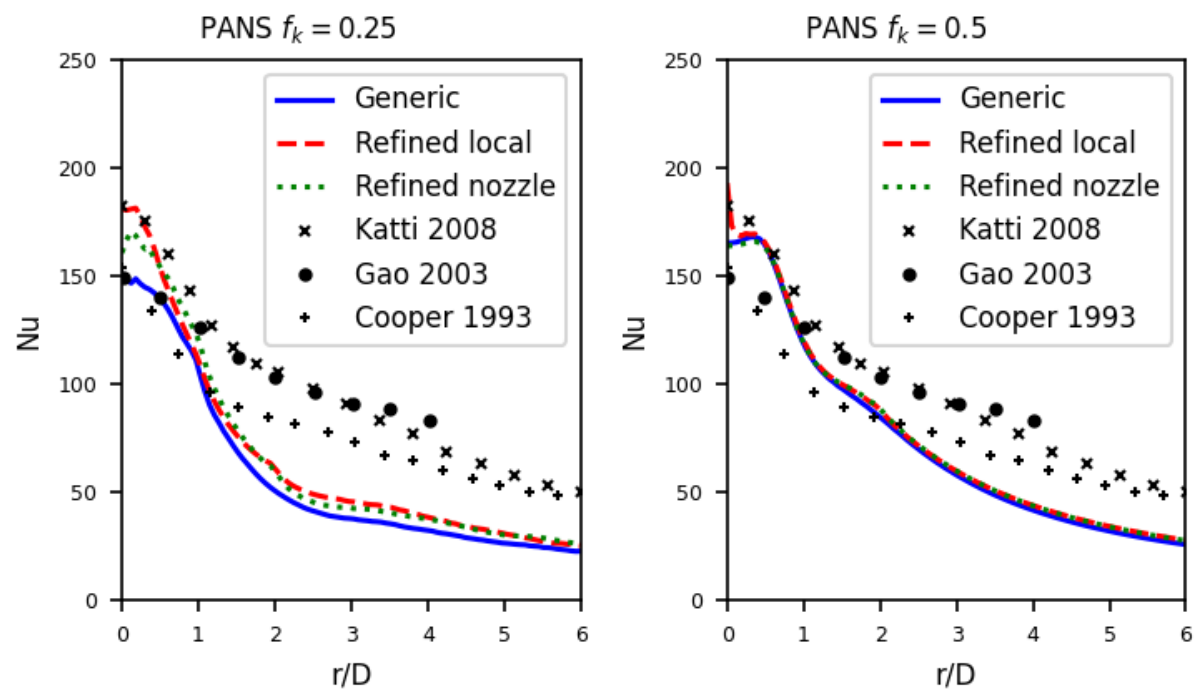

Fig. 6. An influence of the grid refinement on the Nusselt number distribution on the heated wall for PANS models.

An influence of the grid refinement on the eddy viscosity is shown on Figure 7. It is evident that the spatial filtering of DES and LES influences the predicted eddy viscosity and a finer spatial resolution leads to a lower eddy viscosity. In contrary, a minimal influence of the spatial resolution is observed on the eddy viscosity for PANS as the filtering is rather physical than spatial.

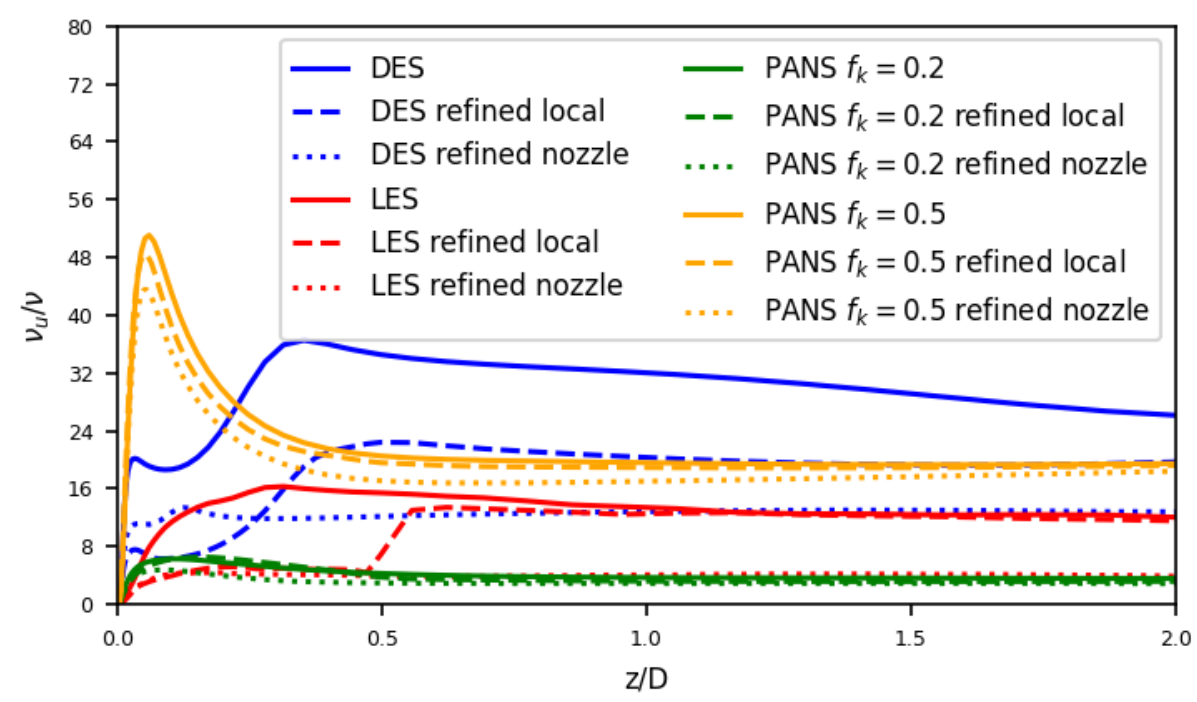

Fig. 7. Ratios of the eddy and molecular viscosity along the nozzle axis (the heated wall at $z / D=0$ ).

Additionally, an influence of the boundary condition applied on the upper surface is investigated in the present work. The influence of the boundary condition is shown on Figure 8 where distributions of the time-averaged Nusselt numbers are displayed. There is 
no difference in results for DES and RANS simulations up to 1 diameter from the nozzle axis, only a slightly higher Nusselt number at the stagnation point can be observed for the DES with no slip condition. Further downstream a minimal difference can be observed. For both methods, the simulation with the slip condition prescribed on the upper surface predicted lower values of the Nusselt number in comparison with other two conditions.
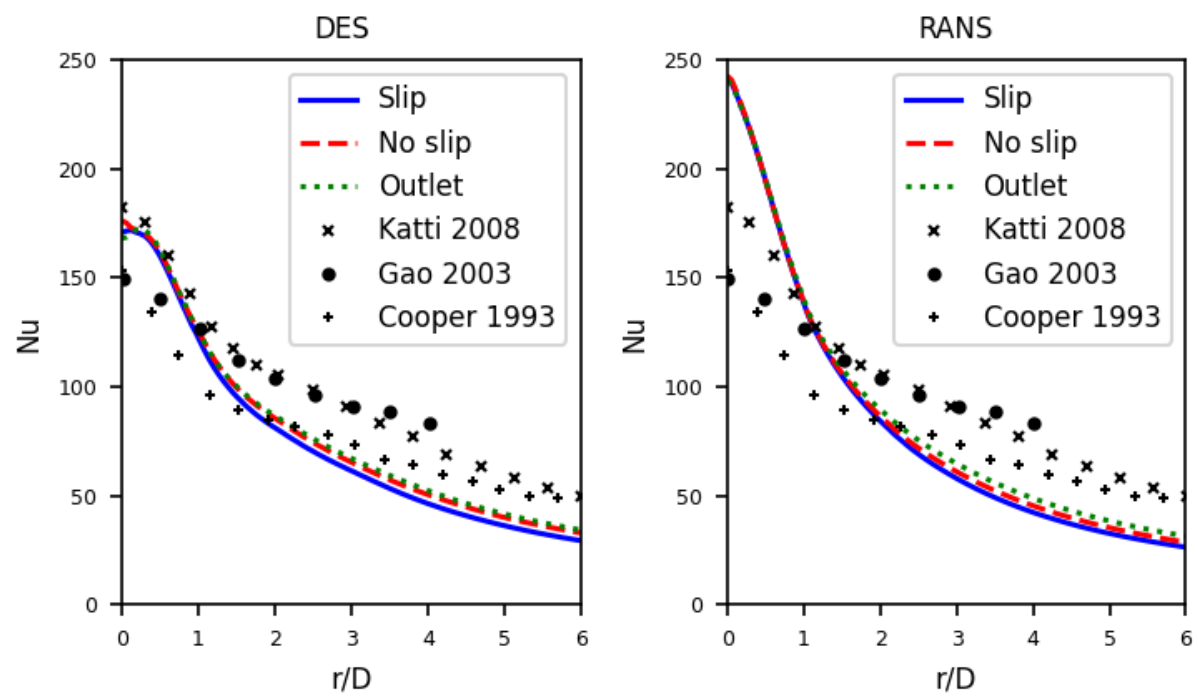

Fig. 8. Influence of the boundary condition on the Nusselt number distribution.

The physical resolution influences predicted flow structures as it is shown on Figure 9 where contours of the instantaneous velocity magnitude are displayed for both PANS simulations $\left(f_{k}=0.25\right.$ and $\left.f_{k}=0.5\right)$. The simulation with the higher physical resolution $\left(f_{k}=0.25\right)$ was able to capture vortex breakdown below the nozzle exit. In contrary, the higher eddy viscosity in the case of PANS simulation with $f_{k}=0.5$ damped almost all introduced velocity fluctuations. Damped fluctuations does not necessary mean a worse prediction of turbulent heat transfer using PANS as they results in a higher eddy viscosity. Additionally, the physical resolution $f_{k}=0.5$ was recently identified as an upper threshold for the correct prediction of transition in the free shear layer [21]. We assume that this upper threshold varies among fluid flow problems and a further analyse on more physical resolutions would be beneficial for a use of PANS for practical applications. 


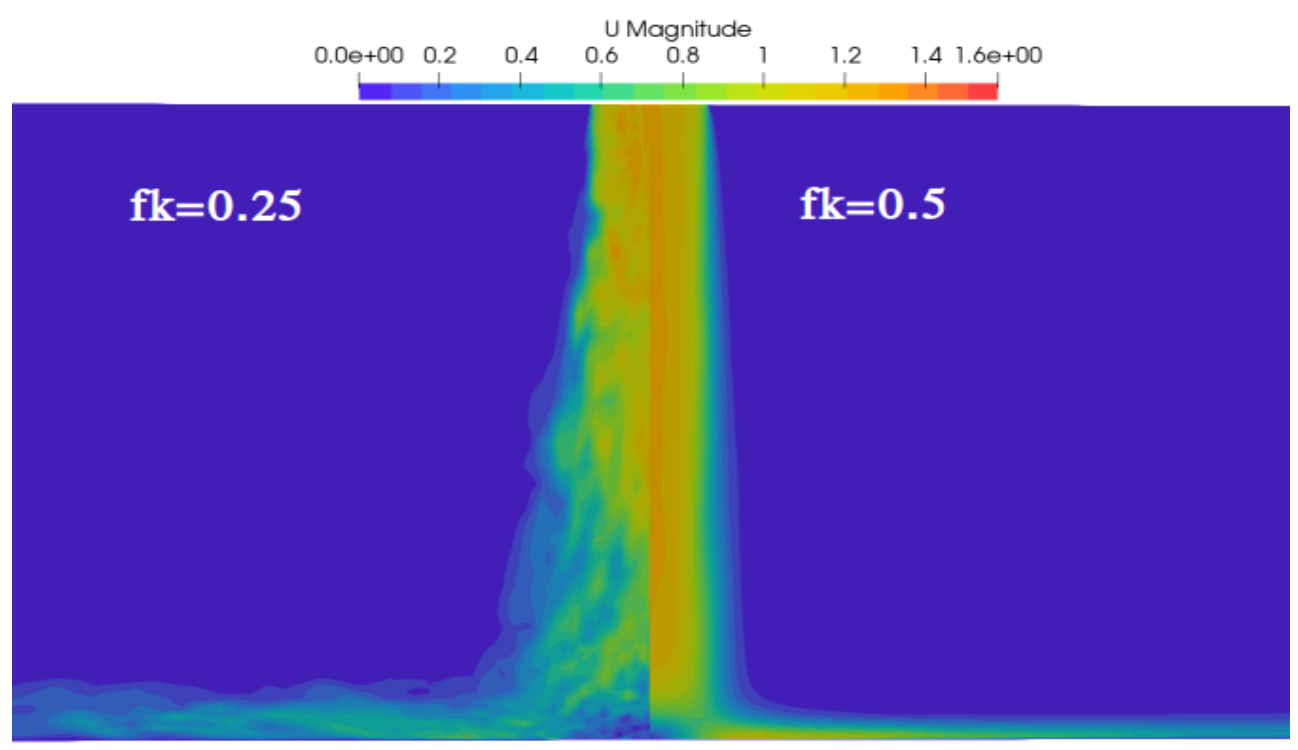

Fig. 9. Contours of the instantaneous velocity magnitude for PANS models.

\section{Conclusions}

The present study aimed on a comparison of SRS methods (DES, LES and PANS) on the impinging jet heat transfer at $R e=23000$ and $H / D=6$ with a closer insight on an influence of a local grid refinement. Results obtained with the DES model and the PANS with the physical resolution $f_{k}=0.5$ were closest to experimental data for all three computational grids. LES and PANS with the physical resolution $f_{k}=0.25$ under-predicted heat transfer far from the nozzle axis $r / D>1$ and we assume that it corresponds to a coarse/insufficient spatial resolution in this region. Both tested grid refinements led to an increase of predicted heat transfer near the stagnation point for LES and PANS $f_{k}=0.25$. Additionally, the RANS k- $\omega$ TNT model was also tested and the model over-predicted heat transfer near the stagnation point up to 1 diameter from the nozzle axis and the grid refinements had no influence on predicted results. Further, an investigation of the boundary condition applied on the upper surface showed that the boundary condition has a minimal impact on the predicted heat transfer from the heated wall.

In summary, the results of the SRS models are in a better agreement with experimental data than those obtained with RANS and the grid refinements improves predictions only for models with a high physical resolution.

This publication was written at the Technical University of Liberec with the support of the Specific University Research Grant No. 21291, as provided by the Ministry of Education, Youth and Sports of the Czech Republic in the year 2020.

Computational resources were supplied by the project "e-Infrastruktura CZ" (e-INFRA LM2018140) provided within the program Projects of Large Research, Development and Innovations Infrastructures. 


\section{References}

1. P. Ranjan, A. Dewan, Int. J. Heat and Mass Transfer, 89 (2015)

2. T. Korinek, K. Frana, Manufacturing Technology, 15 (2015)

3. T. Korinek, K. Frana, Journal of Engineering Thermophysics, 26 (2017)

4. J. W. Baughn, A. E. Hechanova, X. Yan, Journal of Heat Transfer, 113 (1991)

5. J. W. Baughn, S. Shimizu, Journal of Heat Transfer, 111 (1989)

6. V. Katti, S. V. Prabhu, Int. J. Heat and Mass Transfer, 51 (2008)

7. N. Gao, H. Sun, D. Ewing, Int. J. Heat and Mass Transfer, 46 (2003)

8. D. Cooper, D. C. Jackson, B. Launder, G. X. Liao, Int. J. Heat and Mass Transfer, 36 (1993)

9. F. Nicoud, F. Ducros, Flow, Turbulence and Combustion, 62 (1999)

10. M. Lasota, P. Sidlof, Topical Problems of Fluid Mechanics 2020, (2020)

11. M. Strelets, AIAA paper, $\mathbf{x x ~ ( 2 0 0 1 ) ~}$

12. F. Menter, M. Juntz, R.B. Langtry, Heat and Mass Transfer, 4 (2003)

13. S. Girimaji, Journal of Applied Mechanics, 73 (2006)

14. F. S. Pereira, L. Eca, G. Vaz, Journal of Fluids Engineering, 141 (2019)

15. J. Kok, AIAA J., 38 (2000)

16. H. Foroutan, S. Yavuzkurt, Int. J. Heat and Fluid Flow, 50 (2014)

17. T. Korinek, T. Tisovsky, J. Hujer, K. Zimmerman, Topical Problems of Fluid Mechanics 2020, (2020)

18. M. Hadziabdic, K. Hanjalic, Journal of Fluid Mechanics, 596 (2008)

19. S. Kubacki, E. Dick, Int. J. Heat and Fluid Flow, 32 (2011)

20. T. Korinek, T. Tisovsky, Topical Problems of Fluid Mechanics 2019, (2019)

21. F.S. Pereira, L. Eca, G. Vaz, S. S. Girimaji, Journal of Computational Physics, 363 (2018) 